\title{
Stimulated Brillouin scattering from multi-GHz-guided acoustic phonons in nanostructured photonic crystal fibres
}

\author{
P. DAINESE 1,2 , P. ST. J. RUSSELL ${ }^{1,3 *}$, N. JOLY1,3 , J. C. KNIGHT11 , G. S. WIEDERHECKER ${ }^{2}$, H. L. FRAGNITO², \\ V. LAUDE4 ${ }^{4}$ AND A. KHELIF4
}

${ }^{1}$ Photonics \& Photonic Materials Group, Department of Physics, University of Bath, Bath BA2 7AY, UK

${ }^{2}$ CePOF, Instituto de Física, Universidade Estadual de Campinas, 13.083-970 Campinas SP, Brazil

${ }^{3}$ Max-Planck Research Group (IOIP), University of Erlangen-Nuremberg, Guenther-Scharowsky-Str., 1/Bau 24, 91058 Erlangen, Germany

${ }^{4}$ Département LPMO, Institut FEMTO-ST, 32 avenue de l'Observatoire, F-25044 Besançon cedex, France

*e-mail: russell@optik.uni-erlangen.de

W avelength-scale periodic microstructuring dramatically alters the optical properties of materials. An example is glass photonic crystal fibre ${ }^{1}$ (PCF), which guides light by means of a lattice of hollow micro/nanochannels running axially along its length. In this letter, we explore stimulated Brillouin scattering in PCFs with subwavelength-scale solid silica glass cores. The large refractive-index difference between air and glass allows much tighter confinement of light than is possible in all-solid single-mode glass optical fibres made using conventional techniques. When the silica-air PCF has a core diameter of around $70 \%$ of the vacuum wavelength of the launched laser light, we find that the spontaneous Brillouin signal develops a highly unusual multi-peaked spectrum with Stokes frequency shifts in the $10-\mathrm{GHz}$ range. We attribute these peaks to several families of guided acoustic modes each with different proportions of longitudinal and shear strain, strongly localized to the $\operatorname{core}^{2,3}$. At the same time, the threshold power for stimulated Brillouin scattering ${ }^{4}$ increases fivefold. The results show that Brillouin scattering is strongly affected by nanoscale microstructuring, opening new opportunities for controlling light-sound interactions in optical fibres.

Tight confinement of both sound and light strongly enhances acousto-optic interactions ${ }^{2,5-8}$, and is relevant in a range of different phenomena such as all-fibre acousto-optic modulators ${ }^{8}$, stimulated Brillouin scattering ${ }^{4,9}$ and optically controlled slow and fast light ${ }^{10}$. Among these, stimulated Brillouin scattering is the phonon-related nonlinear effect most commonly studied in conventional SMFs. Axially propagating acoustic modes can Bragg-scatter guided laser light into a counter-propagating optical mode, with a Doppler shift equal to the acoustic frequency. In single-mode glass optical fibre (SMF), in which a Ge-doped silica core is surrounded by a pure fused silica cladding, the difference in acoustic properties between the core and cladding is very small and there is little confinement of acoustic energy. Furthermore, coupling between shear (S) and longitudinal $(\mathrm{L})$ waves at the core-cladding interfaces is extremely weak and Brillouin scattering is dominated by L-acoustic quasiplane waves (S-strain does not produce significant modulation of the refractive index). As a result, in SMF the Brillouin spectrum is almost the same as in bulk glass, with a single Brillouin peak corresponding to L-acoustic waves travelling along the fibre axis.

In contrast, in air-glass PCFs with large air-filling fractions and micrometre-sized solid glass cores, the 'hard' glass/air interfaces (the core is held in place by a lattice of fused silica nanowebssee insets of Fig. 1c) couple S and L waves together, and the resulting acoustic modes contain proportions of both shear and dilatational $\operatorname{strain}^{2,3}$. As $\mathrm{L}$ and $\mathrm{S}$ waves travel at widely different phase velocities $\left(c_{\mathrm{L}}=5,970.7 \mathrm{~m} \mathrm{~s}^{-1}\right.$ and $c_{\mathrm{S}}=3,764.8 \mathrm{~m} \mathrm{~s}^{-1}$ in silica), this gives rise to families of hybrid (that is, mixed L \& S) modes with markedly different dispersion relations $\beta_{\mathrm{AC}}(\Omega)$, each with its own Brillouin frequency shift $\Omega / 2 \pi$. Figure 1a shows the measured spectra of the spontaneously back-scattered light in two different PCFs (see the Methods section for experimental details). The larger-core PCF (diameter $9.27 \mu \mathrm{m}$ ) showed just one peak at $11.12 \mathrm{GHz}$, which corresponds to the value obtained in bulk glass (for which the phase-matching condition occurs at $\Omega / 2 \pi=2 \nu_{\mathrm{P}} n_{\mathrm{s}} c_{\mathrm{L}} / c=11.12 \mathrm{GHz}$, where $n_{\mathrm{s}}$ is the refractive index of silica at the pump laser frequency $v_{\mathrm{P}}$ ). In contrast, the smallestcore PCF (core diameter $1.22 \mu \mathrm{m}$ ) yielded a family of distinct peaks at $9.76,9.95$ and $10.22 \mathrm{GHz}$, each related to a different axially propagating acoustic mode.

In this PCF, the scattering was found to be strongly dependent on the polarization state of the light-a consequence of structural anisotropy in the core ${ }^{11}$. The fibre birefringence was measured as $\Delta n=0.0004$, and the principal birefringent axes of the fibre were identified (Fig. 1b). By varying the pump polarization state (using a polarimeter) it was possible to explore the polarization dependence 

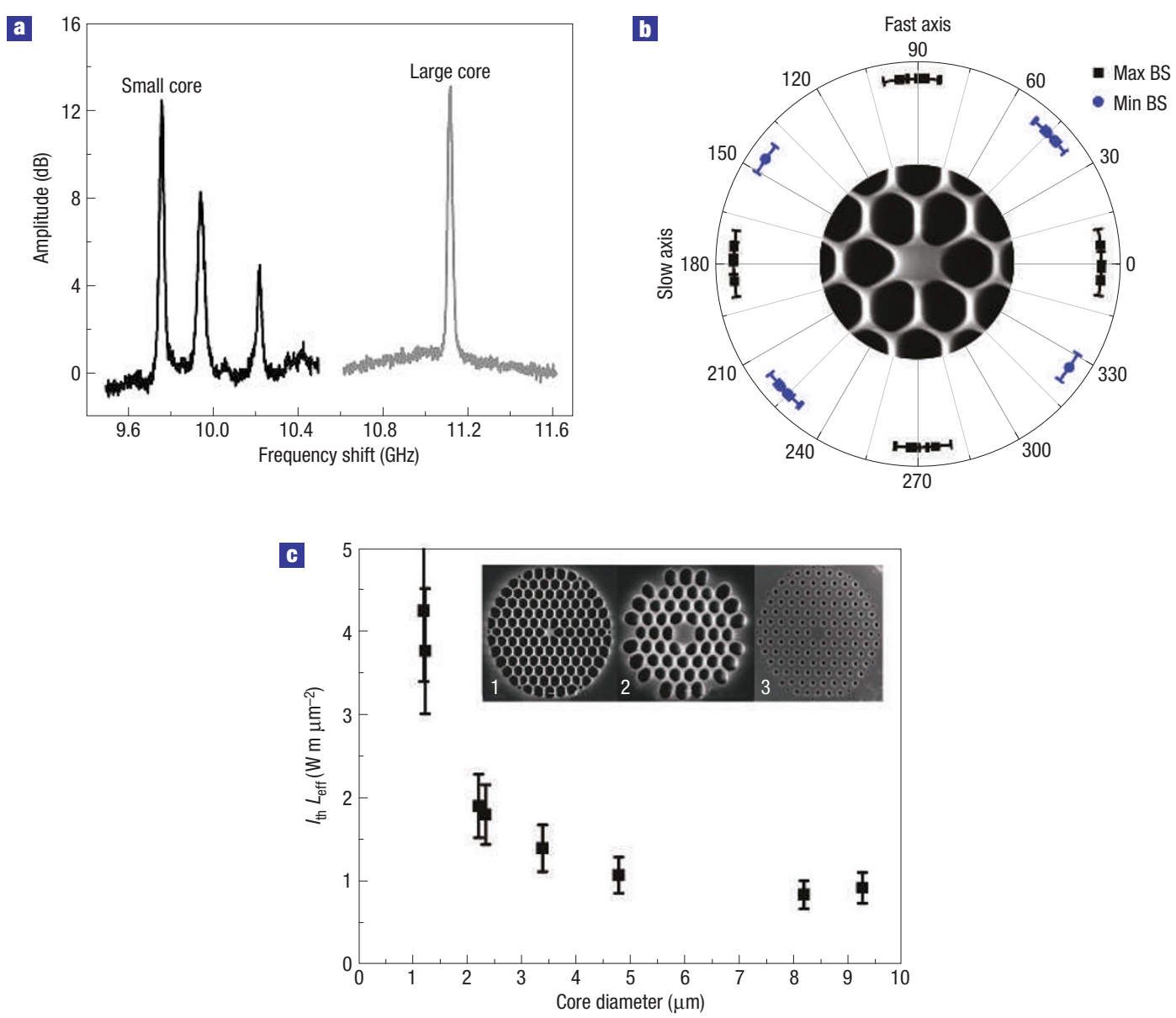

Figure 1 General aspects of Brillouin scattering in small-core photonic crystal fibres. a, Spontaneous Brillouin back-scattering spectra for PCFs with core diameters $1.22 \mu \mathrm{m}$ and $9.27 \mu \mathrm{m}$. The spectra (and also that in Fig. 2) were obtained using a heterodyne detection with $1 \mathrm{MHz}$ of electronic resolution. The vertical scale is logarithmic, and the baseline corresponds to the shot noise level ( $-66 \mathrm{~dB} \mathrm{~m}$ in this case). The large-core fibre yielded scattering at $11.12 \mathrm{GHz}$ with full-width at half-maximum of $31 \mathrm{MHz}$. In contrast, in the small-core case three clear peaks are observed at 9.76, 9.95 and $10.22 \mathrm{GHz}$ with full-width at half-maximum of 24,38 and $25 \mathrm{MHz}$, respectively, for an 84-m-long fibre. A short length (2 m) of the small-core PCF shows the same three peaks but with smaller linewidths: 19,25 and $17 \mathrm{MHz}$, respectively. We attribute this broadening for long fibre to structure fluctuations along the length. $\mathbf{b}$. Characterization of the polarization dependence of back-scattering (BS) in the small-core PCF. The scattered power is maximized when the pump is aligned within the fibre principal axis (slow or fast) and minimized for the pump making $45^{\circ}$ with the axis. The principal states of polarization were characterized using a polarimeter to measure the orientation of the axes with respect to the fibre structure, and short pulses ( $\sim 50 \mathrm{ps)}$ were used to measure the fibre birefringence. The fibre was then cleaved close to the fibre holder and inspected in a microscope at $\times 400$ magnification to identify the orientation of the fast and slow axes relative to the hexagonal cladding structure. The error bars indicate the experimental uncertainty. c, Stimulated Brillouin scattering intensity threshold measurements for PCFs with different core diameters. The squares correspond to experimental data and the error bars were estimated to be $20 \%$, arising from the cut-back method used to measure the coupled input power (see the Methods section). For the smallest-core PCF, the threshold increases by a factor of $\sim 5$ compared with large-core PCFs. Inset: scanning electron micrographs of three typical PCFs used in the experiments, with core diameters 1.22 $\mu \mathrm{m}$ (1), $2.36 \mu \mathrm{m}$ (2) and $9.27 \mu \mathrm{m}$ (3).

of the Brillouin spectrum (Fig. 1b). When the pump laser was polarized parallel to the fast or slow principal axis, the Brillouin back-scattered gain coefficient was twice that obtained when the pump was polarized at $45^{\circ}$ to the fibre axis, in broad agreement with Stolen ${ }^{11}$. When the pump wave was polarized along the slow axis, the frequency shift of the Stokes wave was measured to be 3.5 $\mathrm{MHz}$ larger than that obtained when the pump was aligned along the fast axis. Using the measured value of birefringence, a simple calculation shows that the difference in frequency shift is $\Delta f=\left(2 c_{\mathrm{L}} / \lambda\right) \Delta n=3.1 \mathrm{MHz}$ at $\lambda=1.55 \mu \mathrm{m}$, in good agreement with the experimental value.

The threshold power $P_{\text {th }}$ for stimulated Brillouin scattering, defined as the point at which the transmitted power begins to saturate and a strong back-scattered signal appears, is an important limiting parameter in nonlinear optical devices such as parametric amplifiers and wavelength converters, which require narrow-band high-power laser light in the fibre core ${ }^{12} . P_{\text {th }}$ depends on the effective area $A_{\text {eff }}$ of the optical mode, which determines the threshold intensity $I_{\text {th }}=P_{\text {th }} / A_{\text {eff }}$, and the fibre attenuation $\alpha \mathrm{m}^{-1}$, usually taken account of through an effective length $L_{\text {eff }}=\left(1-\mathrm{e}^{-\alpha L}\right) / \alpha$, where $L$ is the physical fibre length ${ }^{13}$. By factoring out the 'obvious' dependence of threshold power on core size, the product $I_{\text {th }} L_{\text {eff }}$ highlights the effects of acoustic confinement on Brillouin scattering. The results of a series of experiments on many different PCFs, with core diameters in the range 1-10 $\mu \mathrm{m}$, are plotted in Fig. 1c (for the small-core PCFs the threshold was measured with the pump aligned with a principal fibre axis). The effective areas were calculated following 


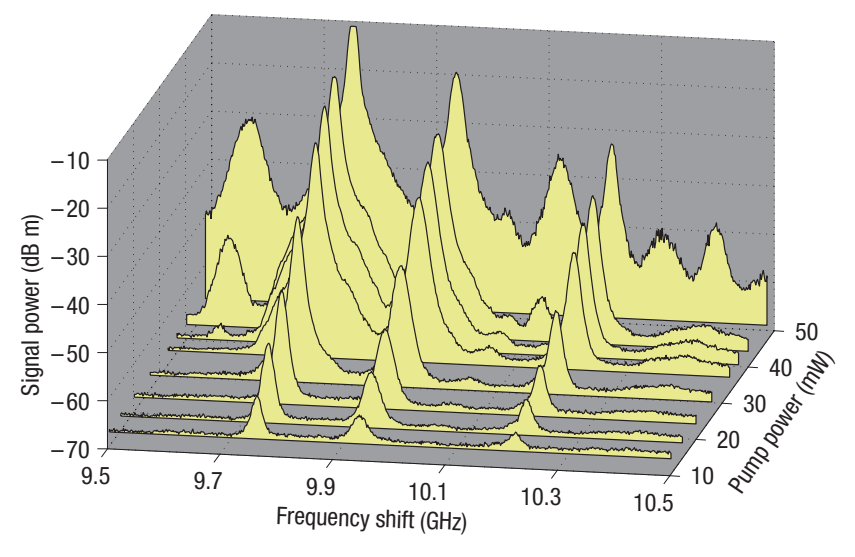

Figure 2 Evolution of the measured Brillouin back-scattering spectrum as a function of the input pump power (in the smallest-core PCF). The vertical scale is logarithmic.

Mortensen ${ }^{14}$ and agreed well with measurements of the numerical aperture. The data shows that $I_{\mathrm{th}} L_{\text {eff }}$ increases as the core diameter decreases, almost independently of the precise microstructure

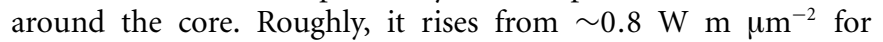
core diameters greater than $8 \mu \mathrm{m}$ to $\sim 4.3 \mathrm{~W} \mathrm{~m} \mu \mathrm{m}^{-2}$ for core diameters $\sim 1 \mu \mathrm{m}$, an enhancement of $7.3 \mathrm{~dB}$. This fivefold increase in threshold power is in striking contrast to standard fibres, where the product $I_{\mathrm{th}} L_{\text {eff }}$ is almost independent of core diameter ${ }^{4}$. This increased threshold can be explained by the complex radial and azimuthal pattern of strain across the core, with strong S-strain components and nodes in the L-strain field (a more complete discussion follows later). Briefly, the presence of shear strain reduces the effective refractive-index perturbation, so that threshold is achieved at higher powers.

We also recorded the evolution of the back-scattered spectrum with pump power, as shown in Fig. 2, for the smallest-core PCF. For high pump powers the spectrum develops more structure and peaks appear at new frequencies. The origin of this spectral broadening is as yet unclear, although it may be related to parametric frequency mixing and conversion of the light or possibly the sound ${ }^{15}$.

It has previously been shown that the optical modes of small-core PCFs (such as those illustrated in Fig. 1) are quite accurately described by the optical modes of a circular-cylindrical strand of silica in vacuum ${ }^{16}$. It is perhaps surprising that the $10-\mathrm{GHz}$ Brillouin band in small-core PCF is also semi-quantitatively described by an acoustic analysis based on a single silica strand ${ }^{17,18}$, despite neglecting the effects of the narrow glass webs around the core. The observed decrease in frequency shift as the core diameter is reduced can be explained by the strong optical and acoustic index contrast between glass and air. The Bragg-scattering condition requires the acoustic propagation constant to be twice the optical propagation constant, $\beta_{\mathrm{AC}}=2 \beta$. Assuming that the optical and L-acoustic modes are perfectly confined to a circular-cylindrical glass strand of radius $a$ surrounded by air, the optical and acoustical transverse wavevectors are approximately given by $k_{\mathrm{T}}=z_{01} / a$, where $z_{01}=2.405$ is the first zero of the Bessel function $J_{0}$. Given that $\beta=\sqrt{\left(\omega_{\mathrm{P}} n_{\mathrm{s}} / c\right)^{2}-k_{\mathrm{T}}^{2}}$ and $\beta_{\mathrm{AC}}=\sqrt{\left(\Omega / c_{\mathrm{L}}\right)^{2}-k_{\mathrm{T}}^{2}}$, where $\omega_{\mathrm{P}}$ is the angular frequency of the pump light, $n_{\mathrm{s}}=1.444$ is the refractive index of silica (at $1.55 \mu \mathrm{m}), c$ is the speed of light in vacuum and $c_{\mathrm{L}}$ is the longitudinal acoustic velocity, the phase-matching condition $\beta_{\mathrm{AC}}=2 \beta$ yields the relationship $\Omega=c_{\mathrm{L}} \sqrt{\left(2 \omega_{\mathrm{P}} n_{\mathrm{s}} / c\right)^{2}-3\left(z_{01} / a\right)^{2}}$ for the angular frequency shift. This predicts frequency shifts of 9.04 and 11.09

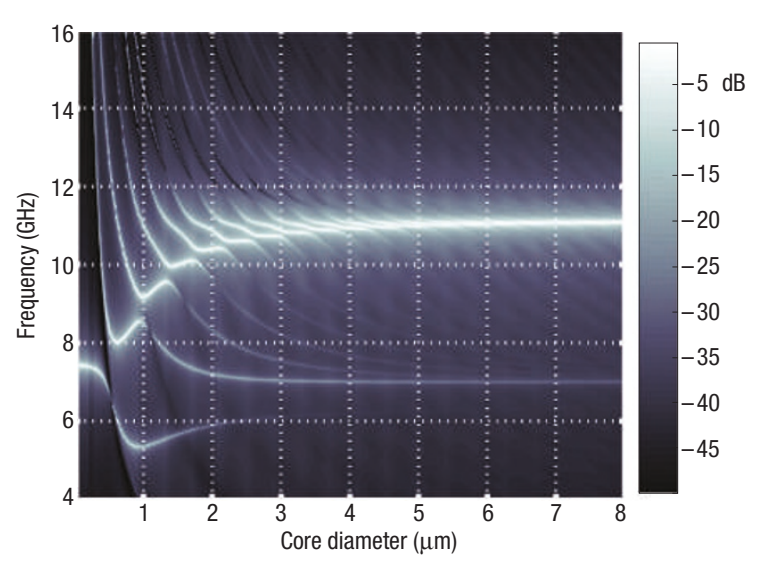

Figure 3 Evolution of the Brillouin backward scattering spectrum as the strand diameter is reduced, showing the splitting. The spectrum was calculated through the evaluation of the overlap between the optical mode with the axial-radial family of acoustic modes. The greyscale shading of the curves represents the strength of the acousto-optical overlap, and for each diameter the calculated spectrum was normalized to the peak value. As the strand gets thinner, the spectrum splits into multiple peaks and the frequency shift of the major peak decreases.

$\mathrm{GHz}$ for the small and large cores respectively-quite good agreement considering the simplicity of the model.

The refractive-index changes induced by a general acoustic strain field can be calculated using the impermeability tensor ${ }^{19}$. For large PCF cores, L-acoustic modes dominate the spontaneous Brillouin spectrum, and a large number of modes, all with very similar dispersion, cluster closely around one frequency. As a result, one dominant band appears in the back-scattered spectrum. However, in very small-diameter strands two things happen. First, the acoustic modes that participate in Brillouin scattering are much more widely spaced in frequency, with strongly dissimilar dispersion relations; and second, they become strongly hybrid, containing large amounts of S-strain. This gives rise to a Brillouin spectrum with several widely spaced peaks. Calculations of the explicit acousto-optic overlap integral for all axial-radial acoustic modes in a circular strand yield the results in Fig. 3, where the evolution of the Brillouin spectrum is plotted against core diameter. The appearance of multiple peaks in the $10-\mathrm{GHz}$ region for small cores is clear, in good qualitative agreement with the experimental observation of three peaks for the smallest-core PCF; also, the frequency shift of the main peak decreases, as predicted in the simple model introduced above. This peak is $25-30 \mathrm{~dB}$ weaker than the main 10-GHz peak and originates from Rayleigh surface waves whose overlap with the optical mode is small. These waves do not appear in bulk glass, and on silica surfaces they propagate with a velocity of approximately $3,410 \mathrm{~m} \mathrm{~s}^{-1}$ (ref. 18), giving a frequency shift of $\sim 7 \mathrm{GHz}$ for light of wavelength $1,550 \mathrm{~nm}$.

Although this simple model is useful as a guide, a complete understanding of the Brillouin spectra rests on a full description of the acoustic modes, which requires careful computation of the actual acoustic modes in the whole PCF structure. We numerically solved the full acoustic wave equation (see the Methods section for details), and calculated the Brillouin spectra (Fig. 4), identifying which modes participate in the Brillouin scattering (movies of a few representative acoustic modes are available in the Supplementary Information). Figure $4 \mathrm{a}$ shows the calculated Brillouin spectrum, with a series of three main peaks at approximately 9.3, 9.5 and 9.7 GHz. This is within a $4 \%$ shift of the measured 9.76, 9.95, 

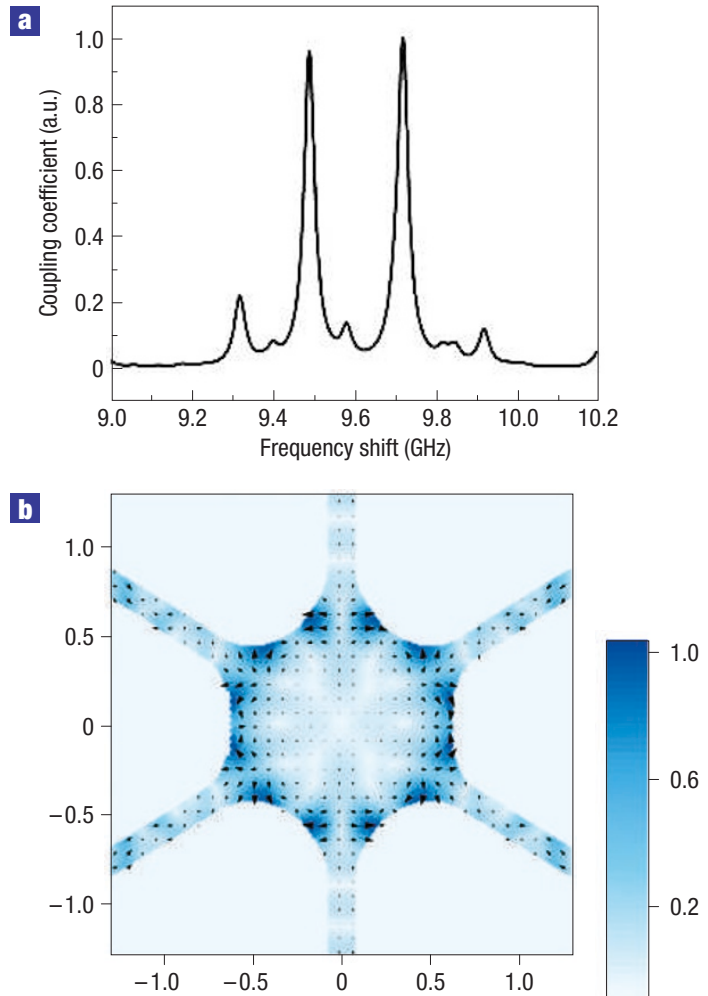

(

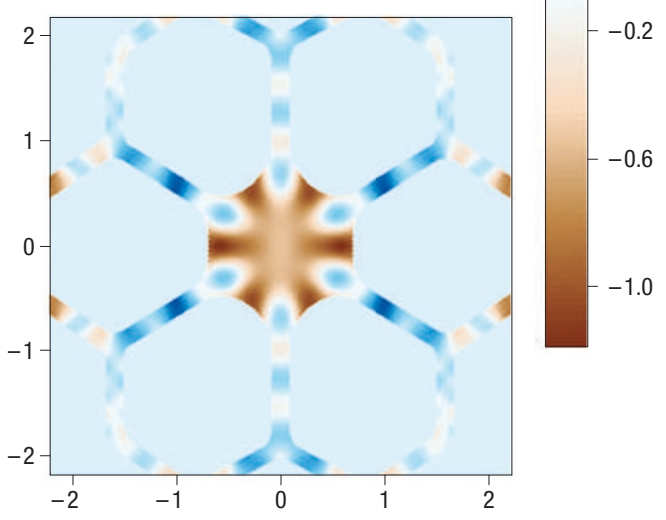

Figure 4 A few representative acoustic modes of the small-core PCF.

a, Calculated acousto-optic coupling coefficient for Brillouin scattering, normalized to the highest peak. $\mathbf{b}$, Longitudinal component and $\mathbf{c}$, transverse component of the acoustic displacement vector for the strongest peak in a. The amplitudes are normalized to unity, and the maximum absolute value of the transverse component was verified to be 0.87 of the longitudinal component.

10.22 $\mathrm{GHz}$ peaks. Considering the uncertainties in the actual fibre dimensions and the silica acoustic constants, there is quite striking agreement. The acoustic displacement vector of this mode is shown in Fig. $4 \mathrm{~b}$ (in-plane displacement) and c ( $z$-components). Nodes in the displacement (responsible for the threshold increase) and the expected sixfold angular symmetry, following the fibre structure, can clearly be seen. The effective acoustic modal area is a good parameter for comparing the threshold of different fibres $^{20}$. Computations yielded a value of $6.1 \mu \mathrm{m}^{2}$, whereas the effective optical modal area was calculated to be $1.4 \mu \mathrm{m}^{2}$. The threshold increase can be roughly estimated as the ratio between the effective acoustic and optical modal areas, giving $10 \log _{10}(6.10 / 1.4)=6.4 \mathrm{~dB}$, in good agreement with the measured value of $7.3 \mathrm{~dB}$. Another contribution to the threshold increase is the strong $z$-component of the electrical field $E_{z}$ in the small-core PCF, calculated to be $\sim 37 \%$ of the transverse component (peak values). The backward and forward optical waves have opposite signs of $E_{z}$, which implies reduced elasto-optic coupling.

In conclusion, the micro/nanostructuring of small-core PCFs gives rise to strong acoustic confinement and substantial changes in the acoustic dispersion relation. This affects the characteristics of Brillouin scattering, the smallest cores exhibiting multiple widely spaced Brillouin back-scattering peaks in the spontaneous spectrum. The ability to manipulate and trap acoustic vibrations at multi-GHz frequencies may also lead to new acousto-optic effects and possibly new classes of ultra-efficient acousto-optic device in which both sound and light are controlled with precision and their interactions enhanced.

\section{METHODS}

\section{EXPERIMENTAL SET-UP}

The Brillouin spectra were measured by launching light from an external-cavity diode laser at $\lambda=1,550 \mathrm{~nm}$ (with a $\sim 100-\mathrm{kHz}$ linewidth) into various PCFs using a pair of microscope lenses. The launched power was carefully calibrated using a cut-back method. The input/output state-of-polarization of the pump laser was measured using a polarimeter. The light scattered in the backward direction was collected using an optical circulator, inserted before the PCF input end, and then amplified using an Er-doped fibre pre-amplifier. A small fraction of the pump laser light was used as reference for heterodyne detection; the signal was detected using a fast $(20 \mathrm{GHz})$ photodiode, amplified using a radiofrequency preamplifier and its spectrum measured in an electrical spectrum analyser with 1-MHz resolution. The threshold power for stimulated Brillouin scattering was defined as the point at which the transmitted power began to saturate; a 2-W Er-doped fibre amplifier was used for these measurements.

\section{EINITE ELEMENT ANALYSIS}

By creating a non-uniform discretization mesh of the small-core PCF structure and solving the full-vectorial acoustic wave equation using finite element techniques $^{21}$, a precise representation of the acoustic modes of the photonic crystal fibres was obtained. The eigen solutions were sought for a given longitudinal wavevector $\beta_{\mathrm{AC}}$, phase-matched with the PCF optical mode (which was also obtained numerically for the same PCF structure). As the structure was finite, a discrete spectrum of acoustic modes resulted. Unlike in a simple silica strand, the complex geometry and 'hard' boundary conditions couple all three displacement components ( $\hat{r}, \hat{\theta}$ and $\hat{z}$ directions), so that no mode is purely $\mathrm{S}$ or $\mathrm{L}$, although one of these can be dominant for a particular mode.

Received 10 April 2006; accepted 28 April 2006; published 21 May 2006.

\section{References}

1. Russell, P. St. J. Photonic crystal fibers. Science 299, 358-362 (2003).

2. Russell, P. St. J., Marin, E., Diez, A. \& Movchan, A. B. Sonic band gaps in PCF preforms: enhancing the interaction of sound and light. Opt. Express 11, 2555-2560 (2003).

3. Laude, V. et al. Phononic band-gap guidance of acoustic modes in photonic crystal fibers. Phys. Rev. $B$ 71, 045107 (2005).

4. Ippen, E. P. \& Stolen, R. H. Stimulated Brillouin scattering in optical fibers. Appl. Phys. Lett. 16, 539-541 (1972).

5. Gorishnyy, T. et al. Hypersonic phononic crystals. Phys. Rev. Lett. 94, 115501 (2005).

6. Russell, P. St. J. Proc. Conf. Nonlinear Optics Vol. 79 377-379 (Optical Society of America, Washington DC, 2002).

7. Trigo, M. et al. Confinement of acoustical vibrations in a semiconductor planar phonon cavity. Phys. Rev. Lett. 89, 227402 (2002).

8. Birks, T. A., Culverhouse, D. \& Russell, P. St. J. The acousto-optic effect in single mode fibre tapers and couplers. J. Lightwave Technol. 14, 2519-2529 (1996).

9. Boyd, R. W. Nonlinear Optics (Academic, San Diego, 1992).

10. Okawashi, Y. et al. Tunable all-optical delays via Brillouin slow light in an optical fiber. Phys. Rev. Lett. 94, 153902 (2005).

11. Stolen, R. H. Polarization effects in fiber Raman and Brillouin lasers. IEEE J. Quantum Electron. 15, $1157-1160$ (1979).

12. Hansryd, J. et al. Fiber-based optical parametric amplifiers and their applications. IEEE J. Sel. Top. Quantum Electron. 8, 506-520 (2002).

13. Smith, R. G. Optical power handling capacity of low loss optical fibers as determined by stimulated Raman and Brillouin scattering. Appl. Opt. 11, 2489-2494 (1972). 


\section{LETTERS}

14. Mortensen, N. A. Effective area of photonic crystal fibers. Opt. Express 10, 341-348 (2002).

15. Jacob, X., Takatsu, R., Barrière, C. \& Royer, D. Experimental study of the acoustic radiation strain in solids. Appl. Phys. Lett. 88, 134111 (2006).

16. Knight, J. C. et al. Anomalous dispersion in photonic crystal fibres. IEEE Photonics Technol. Lett. 12, 807-809 (2000).

17. Waldron, R. A. Some problems in the theory of guided microsonic waves. IEEE Trans. Microwave Theory Techn. 17, 893-904 (1969).

18. Thurston, R. N. Elastic waves in rods and clad rods. J. Acoust. Soc. Am. 64, 1-37 (1978)

19. Yariv, A. \& Yeh, P. Optical Waves in Crystals (Wiley, New York, 1984),

20. Kobyakov, A. et al. Design concept for optical fibers with enhanced SBS threshold. Opt. Express 13, 5338-5346 (2005).

21. Aalami, B. Waves in prismatic guides of arbitrary cross-section. J. Appl. Mech. 40, 1067-1077 (1973).

\section{Acknowledgements}

The work at Bath was supported by the UK Engineering \& Physical Sciences Research Council

(EPSRC), which also funded P. Dainese's visit to the UK through a Platform Grant. The work in Brazil

was funded by the State of São Paulo Research Foundation (Fapesp) and Padtec S. A.

Correspondence and requests for materials should be addressed to P.St.J.R.

Supplementary Information accompanies this paper on www.nature.com/naturephysics.

Competing financial interests

The authors declare that they have no competing financial interests.

Reprints and permission information is available online at http://npg.nature.com/reprintsandpermissions/ 